\title{
Ozaki Procedure for the treatment of Aortic Valve Endocarditis : A Life-saving Alternative
}

\author{
Harun Arbatli ${ }^{1}$, Ali Kubilay Korkut ${ }^{2}$, Okan Coskun ${ }^{1}$, and Cem Alhan ${ }^{3}$ \\ ${ }^{1}$ Hizmet Hospital (Memorial) \\ ${ }^{2}$ Halic University \\ ${ }^{3}$ Acibadem Hospitals Group
}

February 6, 2021

\begin{abstract}
Infective endocarditis (IE) still has a high risk of mortality and morbidity, despite of developments in medical treatment. Intracranial hemorrhage is one of serious complication, which has poor outcome. We present a 30-year-old male with diagnosis of IE. He had severe aortic regurgitation and oscillating vegetations on both side of the aortic valve. Although there was no neurological symptom in preoperative period, cranial magnetic resonance imaging (MRI) revealed millimetric size ischemic lesions at the right cerebral hemisphere. We performed Ozaki procedure. However, cerebral hemorrhage occurred on the first postoperative day. Computerized tomography (CT) revealed hematoma in parietal and occipital lobes. He was treated with conservative anti-edema therapy and discharged from the hospital on the 25th day with minor visual defect of his eye. Echocardiography control revealed mild aortic regurgitation without any other pathologic finding after 18 months. Aortic neocuspidization by using using of glutaraldehyde treated autologous pericardium decreases the risk of fibrosis and calcification, and thrombosis events. Possibility of suspending the anticoagulant and antiplatelet regimen is a significant advantage in this type of repair. Ozaki procedure might be the first-choice surgical reconstruction technique in anatomically suitable IE cases.
\end{abstract}

Ozaki Procedure for the treatment of Aortic Valve Endocarditis :

\section{A Life-saving Alternative}

Harun Arbatlı*, Ali Kubilay Korkut**, Okan Coskun*, Cem Alhan***

* Hizmet Hospital (Memorial), Dept.of Cardiovasc. Surg., Istanbul, Turkey

** Halic University Med. Fac., Dept.of Cardiovasc. Surg., Istanbul, Turkey

*** Acibadem University Med. Fac., Dept.of Cardiovasc. Surg., Istanbul, Turkey

Corresponding

Ali Kubilay Korkut, MD

Halic University Med. Fac.

Sütlüce Mah. Imrahor Cad. No.82, Beyoglu, Istanbul, Turkey

Tlf. 0-90-532 3368277

e.mail. kubilaykrkt@gmail.com

Word count. 1358

Abstract 
Infective endocarditis (IE) still has a high risk of mortality and morbidity, despite of developments in medical treatment. Intracranial hemorrhage is one of serious complication, which has poor outcome.

We present a 30-year-old male with diagnosis of IE. He had severe aortic regurgitation and oscillating vegetations on both side of the aortic valve. Although there was no neurological symptom in preoperative period, cranial magnetic resonance imaging (MRI) revealed millimetric size ischemic lesions at the right cerebral hemisphere. We performed Ozaki procedure. However, cerebral hemorrhage occurred on the first postoperative day. Computerized tomography $(\mathrm{CT})$ revealed hematoma in parietal and occipital lobes. He was treated with conservative anti-edema therapy and discharged from the hospital on the $25^{\text {th }}$ day with minor visual defect of his eye. Echocardiography control revealed mild aortic regurgitation without any other pathologic finding after 18 months. Aortic neocuspidization by using using of glutaraldehyde treated autologous pericardium decreases the risk of fibrosis and calcification, and thrombosis events. Possibility of suspending the anticoagulant and antiplatelet regimen is a significant advantage in this type of repair. Ozaki procedure might be the first-choice surgical reconstruction technique in anatomically suitable IE cases.

Key words. Infective endocarditis, aortic valve endocarditis, Ozaki procedure

Introduction

IE is a deadly, and despite improvements in its management, remains associated with high mortality and morbidity. Neurological complications occur in $20-40 \%$ of patients during IE and have been related with poorer outcome (1). Vegetation size [?] 3cm, mitral valve involvement, S. aureus as the causal pathogen and anticoagulant treatment are risk factors associated with neurological complications (2). Tornos et al. proved in their study that in left-sided IE due to S aureus anticoagulant therapy is strongly correlated with death to neurologic damage, which as a rule occurs early in the course of the disease (3). We present an aortic valve involved IE in a young male patient, who occurred intracranial hemorrhage following Ozaki procedure. Approval by the Institutional Review Board and informed consent were waived according to our institutional policies for anonymized case reports.

Case Report

A 30-year-old male patient was admitted with the diagnosis of aortic valve endocarditis. He had shortness of breath and his functional capacity was in NHYA class III. Second degree AV block (Mobitz II) was seen on electrocardiography. Transthoracic and transesophageal echocardiography revealed a bicuspid aortic valve with severe regurgitation, an oscillating vegetation with $0.8 \times 1.1 \mathrm{~cm}$ diameter located on the aortic side of the non-coronary cusp, and mild mitral regurgitation (Figure 1a and Figure 1b). Although the patient was asymptomatic neurologically, cranial diffusion MRI revealed millimetric size ischemic lesions at the right cerebral hemisphere.

Blood cultures were negative. Ampicillin/sulbactam (2g ampicillin $+1 \mathrm{~g}$ sulbactam iv q6hr) and gentamicin $(3 \mathrm{mg} / \mathrm{kg} /$ day iv q12hr) was given for empiric antibiotic treatment. Furosemide (20mg iv q12hr) and dopamine perfusion $(3 \mu \mathrm{g} / \mathrm{kg} / \mathrm{min}$ iv) were started. Congestive heart failure symptoms were improved in the first two weeks, but severe aortic regurgitation persisted, and a complete AV block occurred for a short-time period no need for pace-maker, urging us for surgery.

Operative technique and postoperative period

Standart median sternotomy, aortic and two-stage right atrial cannulation was carried out. Moderate hypothermia and extracellular crystalloid cardioplegia were used for myocardial protection. The aortic valve was bicuspid, and the non-coronary cusp had ruptured. Both cusps and all the infected annular tissue were excised. There was no abscess formation. Ozaki procedure was performed by using glutaraldehyde treated autologous pericardium (4) (Figure 2a and Figure 2b). Perioperative trans-esophageal echocardiography revealed mild aortic regurgitation.

While weaning from the ventilator; visual agnosia and right sided hemiparesis were recognized. CT revealed intracranial hematoma in the parietal and occipital lobes (Figure 3). Neurological symptoms improved 
following anti-edema therapy. $3 \% \mathrm{NaCl}$ infusion was given to achieve a blood sodium concentration of 145 $155 \mathrm{mEq} / \mathrm{L}$. The patient was discharged on the postoperative $25^{\text {th }}$ day. All motor functions of the right upper extremity recovered in six months; however, he still has a minor visual defect of the left eye.

Aortic tricuspid valve reconstruction (Figure 4a) and mild aortic regurgitation (Figure 4b) were seen on trans-thoracic echocardiography at postoperative $18^{\text {th }}$ month.

Discussion

Despite improvements in medical and surgical treatment, long term mortality and morbidity of IE still remain high (5). Surgical intervention is considered necessary and has significantly improved the survival especially in patients with cardiac failure, drug resistant infections, persistent vegetations, recurrent embolic events, and prosthetic valve endocarditis (5). Furthermore, prognosis is better in case of early surgical intervention, before cardiac tissue destruction and deterioration occurs (5).

Neurological complications occur in $20 \%$ to $40 \%$ of IE patients, and a predict a poor clinical outcome (2). Cessation of anticoagulation should be considered in concomitant bleeding (3).

Valve replacement or preferably valve repair is necessary in aortic valve IE patients. The use of artificial materials should be avoided as much as possible. Stentless valves, aortic homografts or the Ross procedure may be considered in cases with periannular supporting tissue destruction (5). However, especially in younger patients, early degeneration of bioprosthesis occurs due to fibrosis, calcification or immune reactions (6).

Ozaki and coworkers defined an aortic valve reconstruction technique using autologous pericardium treated with glutaraldehyde (7). In this technique all leaflets are resected, and new pericardial leaflets are reconstructed separately for each coronary cusp. Treatment of this pericardial tissue with glutaraldehyde has been shown to decreases the risk of fibrosis and calcification, and thrombo-embolic events in the long term.

The choice of Ozaki procedure enabled us to avoid anticoagulant and antiplatelet therapy, which gave the best chance of survival in IE patient complicated with a postoperative hemorrhagic cerebrovascular event.

Conclusion

We assume that the Ozaki procedure should be the first-choice surgical reconstruction technique in anatomically suitable IE cases. Long term results of the Ozaki procedure are promising with 12 years of follow-up (8).

Acknowledgements

We would like to thank scrub-nurse Mrs. Leyla Kılıç for her support during the whole surgical procedure.

Conflict of Interests

All the authors declare that there is no conflict of interests.

References

1. Heiro M, Nikoskelainen J, Engblom E, Kotilainen E, Marttila R, Kotilainen P. Neurological manifestations of infected endocarditis: a 17-year experience in a teaching hospital in Finland. Arch Intern Med. 2000;160:2781-2787.

2. Garcia-Cabrera E, Fernandez-Hidalgo N, Almirante B, Ivanova-Georgieva R, Noureddine M, Plata A. et.al. Neurological Complications of Infective Endocarditis. Risk Factors, Outcome, and Impact of Cardiac Surgery: A Multicenter Observational Study. Circulation 2013;127:2272-2284.

3. Tornos P, Almirante B, Mirabet S, Permanyer G, Pahissa A, Soler-Soler J. Infective Endocarditis Due to Staphylococcus aureus: Deleterious Effect of Anticoagulant Therapy. Arch Intern Med. 1999;159(5):473475.

4. Ozaki S, Kawase I, Yamashita H, Uchida S, Takatoh M, Kiyohara N. Midterm outcomes after aortic valve neocuspidization with glutaraldehyde-treated autologous pericardium. J Thorac Cardiovasc Surg. 2018 Jun;155(6):2379-2387. 
5. Netzer RO, Altwegg SC, Zollinger E, Tauber M, Carrel T, Seiler C. Infective endocarditis: Determinants of long term outcome. Heart, 2001;88:61-66.

6. Khan SS, Trento A, DeRobertis M, Kass RM, Sandhu M, Czer LS et al. Twenty-year comparison of tissue and mechanical valve replacement. J Thorac Cardiovasc Surg. 2001;122:257-269.

7. Alhan C. Ozaki procedure. Turkish J Thorac Cardiovasc Surg 2019;27(4):451-453.

8. Ozaki S. Ozaki procedure: 1100 patients with up to 12 years of follow-up. Turkish J Thorac Cardiovasc Surg 2019:27(4):454.

Figures

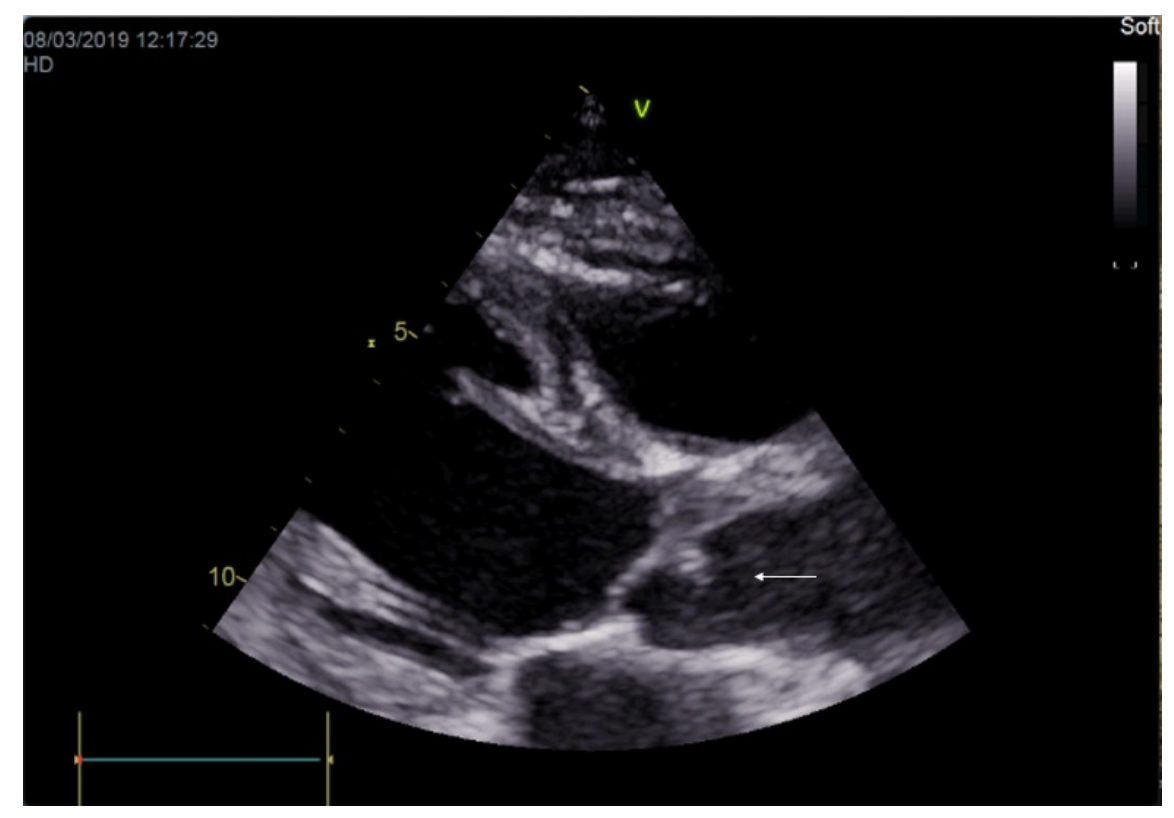

Figure 1a. Transthoracic echocardiography revealed the vegetation located on the aortic side of the noncoronary cusp of a bicuspid aortic valve (white arrow) 


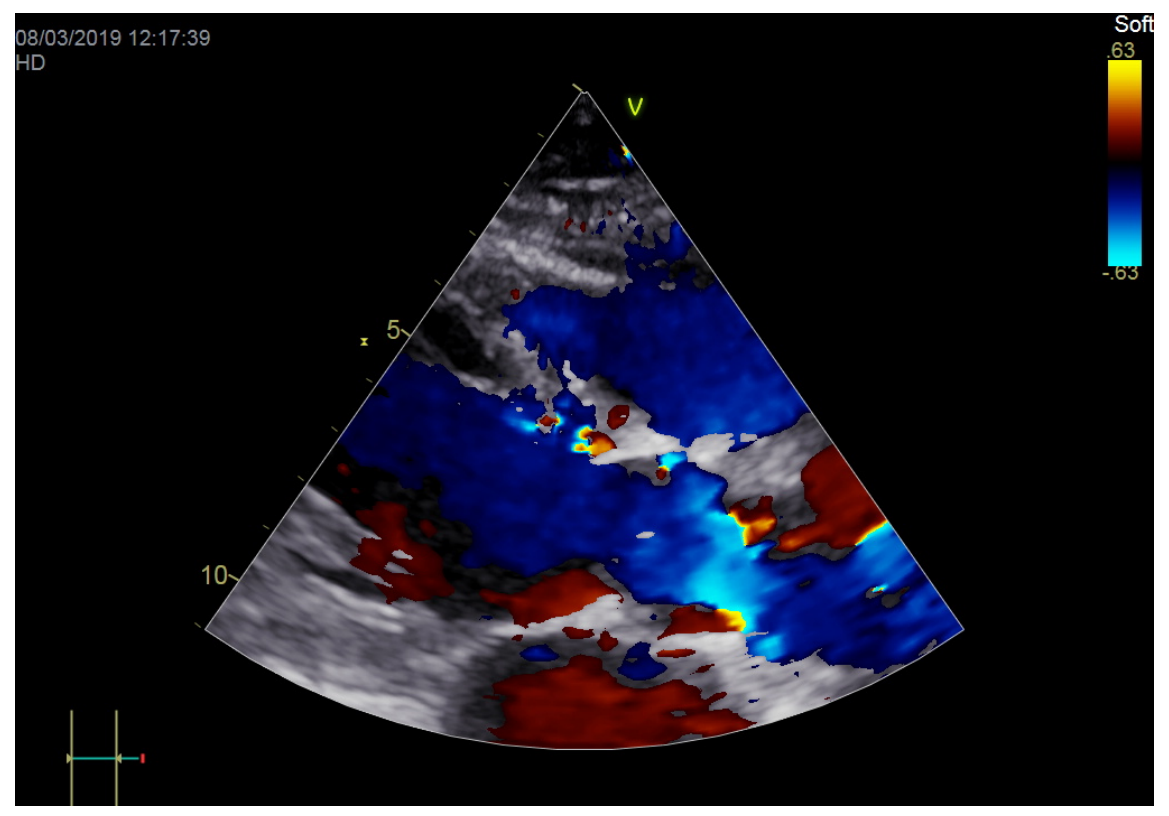

Figure 1b. Aortic valve with severe regurgitation is seen on preoperative transthoracic echocardiography. 


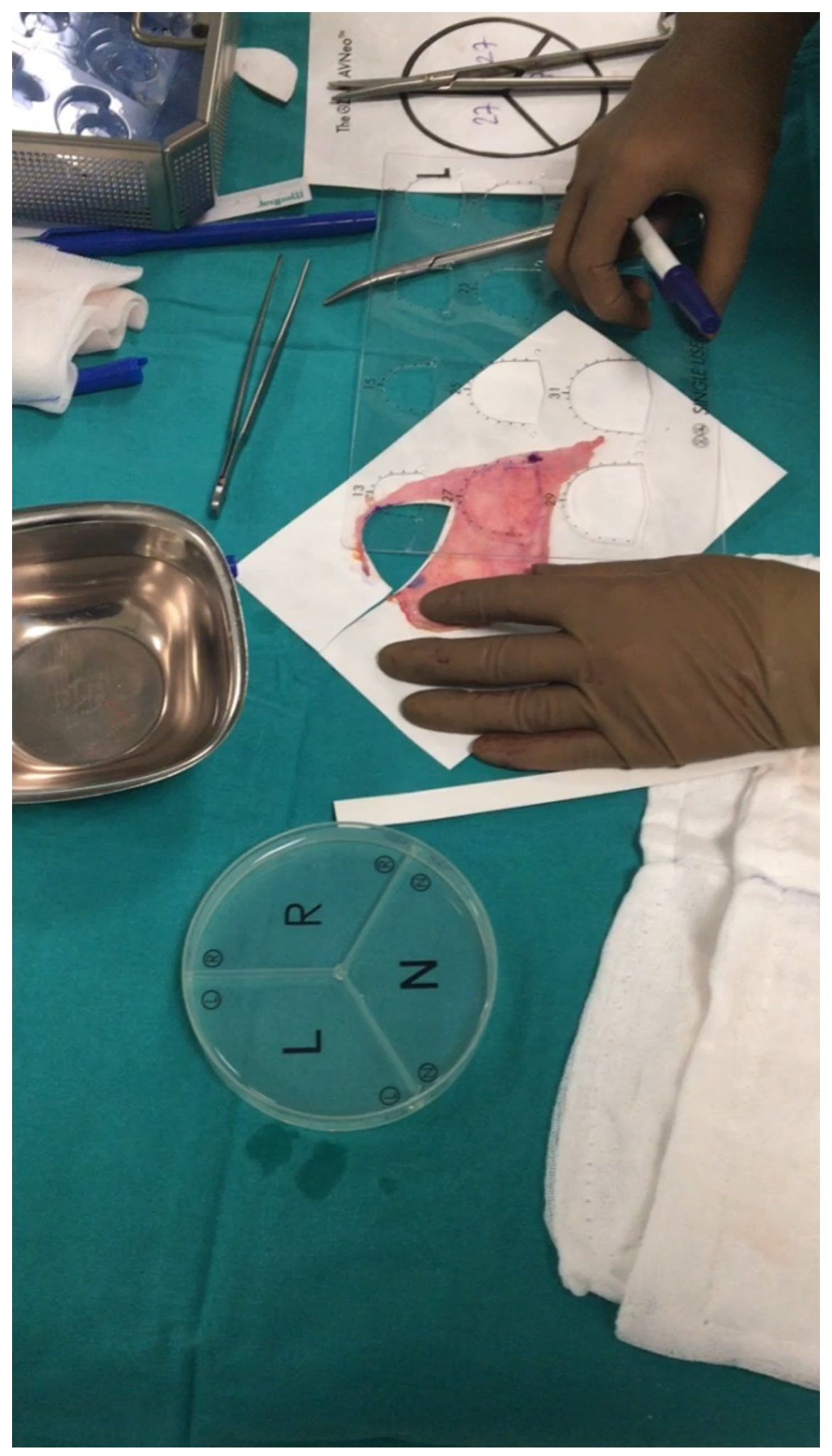

Figure 2a. Neo-aortic cusps are reconstructed using glutaraldehyde-treated autologous pericardium 


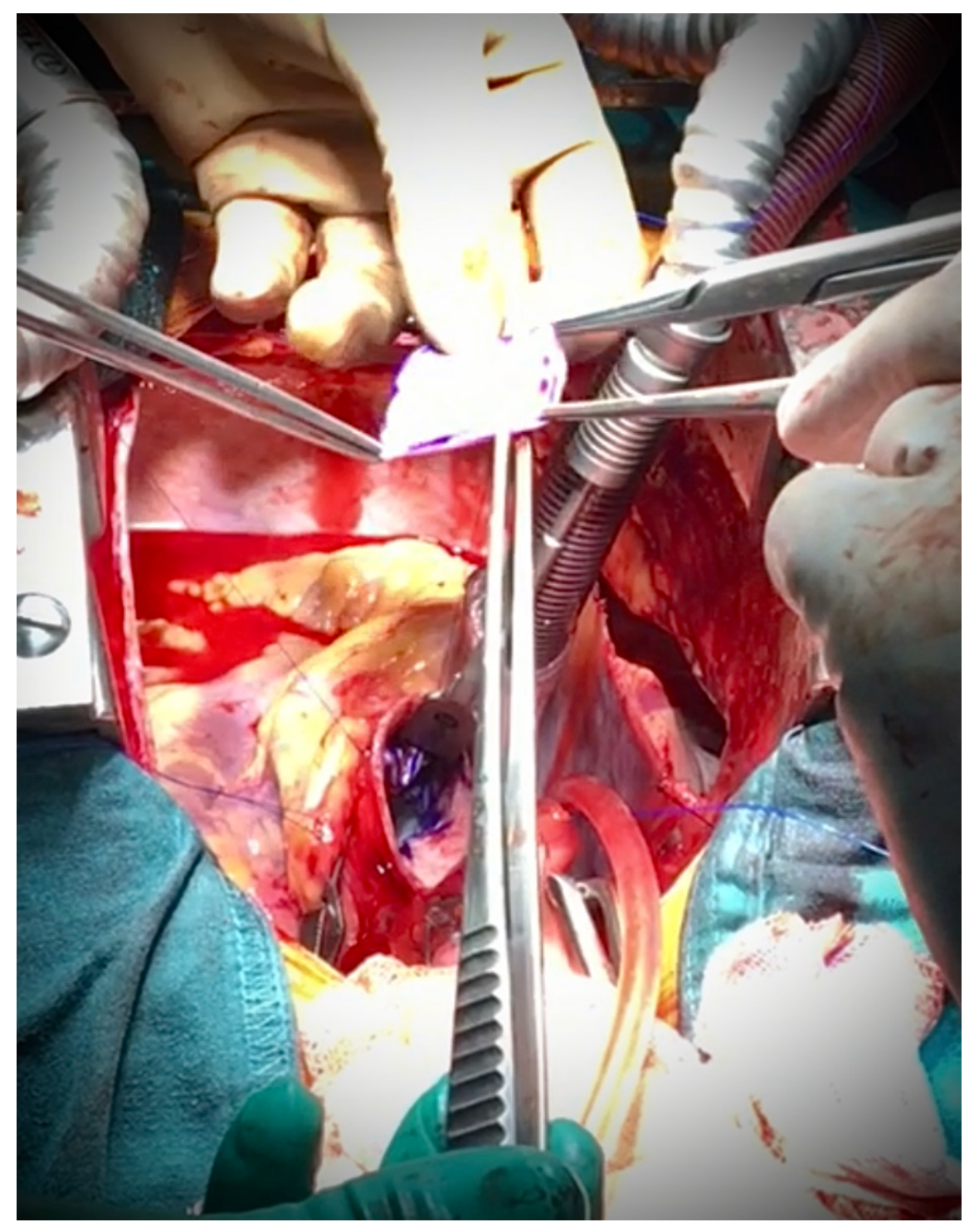

Figure 2b. Pericardial cusps are sutured to the aortic annulus. 


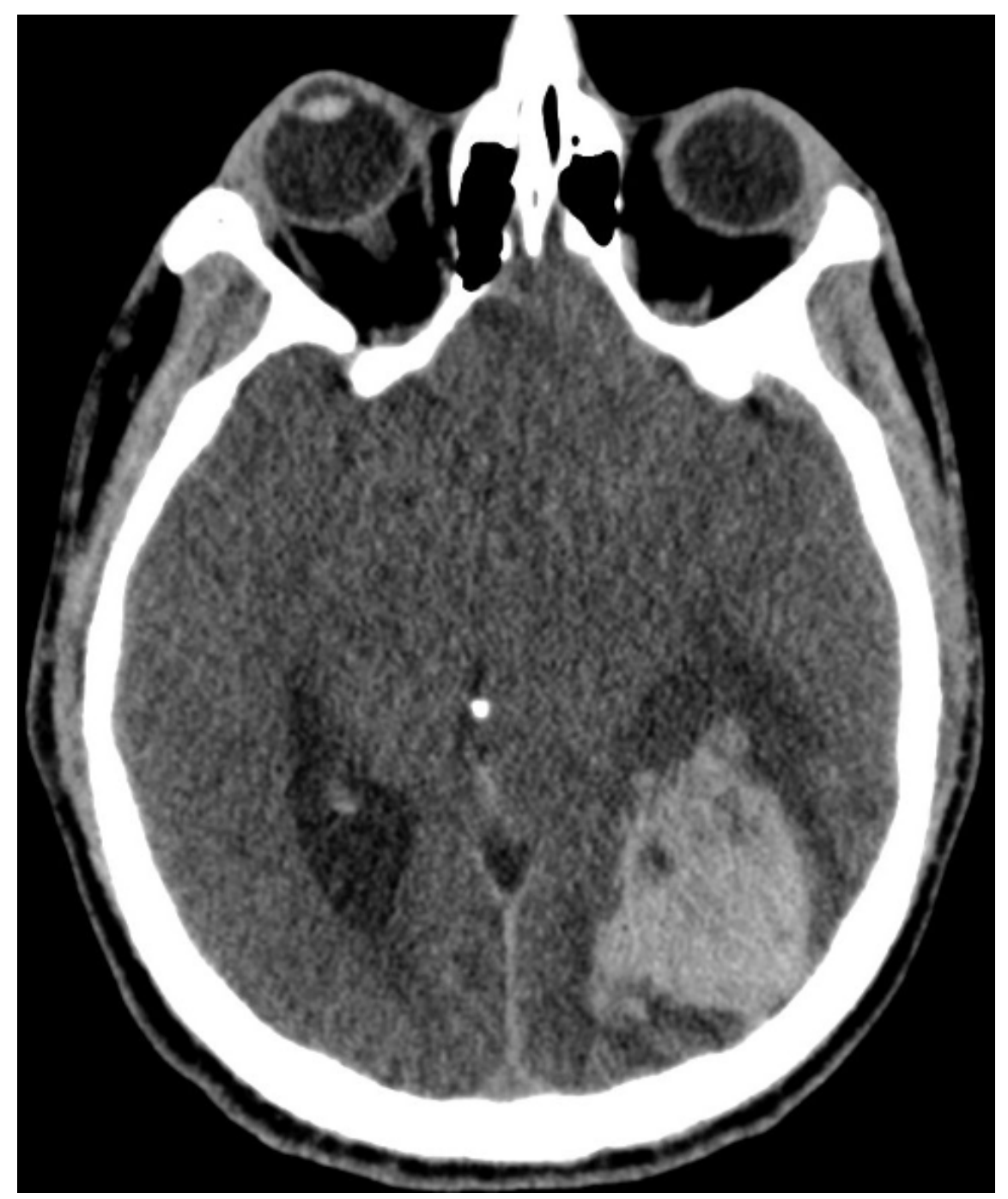

Figure 3. CT revealed intracranial hematoma in the left parietal and occipital lobes. 


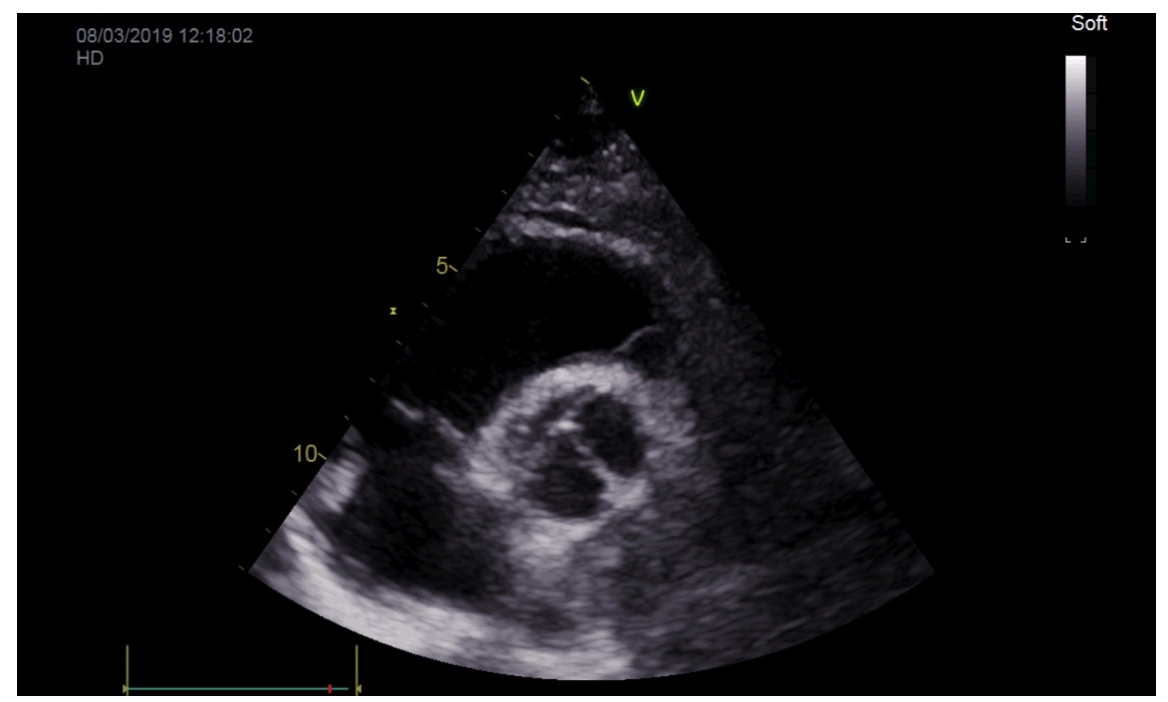

Figure 4a. Aortic tricuspid valve reconstruction is seen on postoperative transthoracic echocardiography.

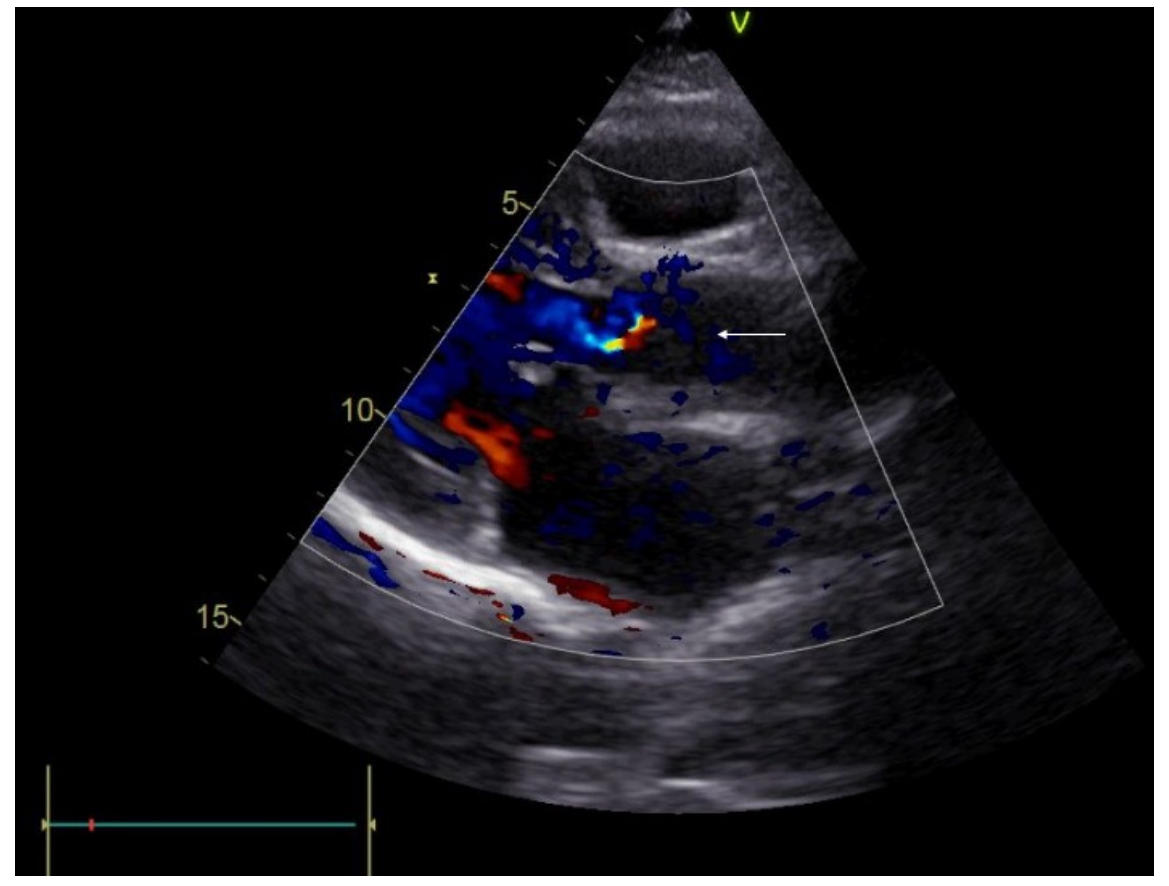

Figure 4b. Transthoracic echocardiography revealed mild regurgitation on postoperative $18^{\text {th }}$ month (white arrow).

\section{Hosted file}

Ozaki procedure Figures.pdf available at https://authorea.com/users/394116/articles/507574ozaki-procedure-for-the-treatment-of-aortic-valve-endocarditis-a-life-saving-alternative 